УДК 517.547

\author{
A. I. BANDURA
}

\title{
ANALYTIC FUNCTIONS IN THE UNIT BALL OF BOUNDED VALUE $L$-DISTRIBUTION IN DIRECTION
}

\begin{abstract}
A. I. Bandura. Analytic functions in the unit ball of bounded value L-distribution in a direction, Mat. Stud. 49 (2018), 75-79.

We present a generalization of concept of bounded value $L$-distribution in direction $\mathbf{b} \in$ $\mathbb{C}^{n} \backslash\{0\}$ for analytic functions in the unit ball $\mathbb{B}^{n} \subset \mathbb{C}^{n}$, where $L: \mathbb{B}^{n} \rightarrow \mathbb{R}_{+}$is a continuous function. It is established a connection between the class of analytic functions in the unit ball of bounded $L$-index in the direction $\mathbf{b}$ and the class of analytic functions in the unit ball of bounded value $L$-distribution in the same direction. The main result of the paper is the following: An analytic function $F: \mathbb{B}^{n} \rightarrow \mathbb{C}$ is a function of bounded value $L$-distribution in a direction $\mathbf{b} \in \mathbb{C}^{n} \backslash\{0\}$ if and only if its directional derivative $\frac{\partial F}{\partial \mathbf{b}}$ has bounded $L$-index in the same direction $\mathbf{b}$.
\end{abstract}

An entire function $f: \mathbb{C} \rightarrow \mathbb{C}$ is called a function of bounded value distribution $[6,8,11$, $12,14]$, if there exist constants $p \geq 0, R>0$ such that the equation $f(z)=w$ for every $w \in \mathbb{C}$ has at most $p$ solutions in any disc of radius $R$.

The function of bounded value distributionhave many interesting properties and applications. It attracted the attention of many mathematicians. Among many interesting and amazing properties of these functions there is the following result obtained by W. Hayman [8]: an entire function has bounded index if and only if its derivative is a function of bounded value distribution. Note [10] that an entire function $f$ is said to be of bounded $l$-index if there exists an integer $m$, independent of $z$, such that for all $p$ and all $z \in \mathbb{C}$ $\frac{\left|f^{(p)}(z)\right|}{p !} \leq \max \left\{\frac{\left|f^{(s)}(z)\right|}{s !}: 0 \leq s \leq m\right\}$.

Later there were introduced the concepts of entire function of bounded valued $l$-distribution and bounded $l$-index [9]. Besides, the mentioned property was generalized by M. Sheremeta for entire functions of bounded $l$-index ([15]).

In the multidimensional case there is a concept of bounded $L$-index in direction for entire functions and for functions analytic in the unit ball $[1-3,5]$. Here we generalize Sheremeta's result ([15]) for analytic functions in the unit ball of bounded value $L$-distribution in direction. Earlier, we deduced this result for entire functions of bounded $L$-index in direction ([4]).

Let $\mathbf{0}=(0, \ldots, 0), \mathbf{b}=\left(b_{1}, \ldots, b_{n}\right) \in \mathbb{C}^{n} \backslash\{\mathbf{0}\}$ be a given direction, $\mathbb{R}_{+}=(0,+\infty)$, $\mathbb{B}^{n}=\left\{z \in \mathbb{C}^{n}:|z|<1\right\}, L: \mathbb{B}^{n} \rightarrow \mathbb{R}_{+}$be a continuous function such that for all $z \in \mathbb{B}^{n}$

$$
L(z)>\frac{\beta|\mathbf{b}|}{1-|z|}, \beta=\text { const }>1
$$

2010 Mathematics Subject Classification: 30D15, 30D30, 34M05.

Keywords: analytic function; unit ball; bounded index; bounded value distribution. doi:10.15330/ms.49.1.75-79

(C) A. I. Bandura, 2018 
For a given $z \in \mathbb{B}^{n}$ we denote $S_{z}=\left\{t \in \mathbb{C}: z+t \mathbf{b} \in \mathbb{B}^{n}\right\}$. Notice that if $\eta \in[0, \beta], z \in \mathbb{B}^{n}$ and $|t| \leq \frac{\eta}{L(z)}$ then $z+t \mathbf{b} \in \mathbb{B}^{n}$. Indeed, we have $|z+t \mathbf{b}| \leq|z|+|t \mathbf{b}| \leq|z|+\frac{\eta|\mathbf{b}|}{L(z)}<|z|+\frac{\beta|\mathbf{b}|}{\frac{\beta \beta}{1-|z|}}=1$.

Analytic function $F: \mathbb{B}^{n} \rightarrow \mathbb{C}$ is called a function of bounded L-index in a direction $\mathbf{b}$ ([5]) if there exists $m_{0} \in \mathbb{Z}_{+}$such that for every $m \in \mathbb{Z}_{+}$and every $z \in \mathbb{B}^{n}$ the following inequality is valid

$$
\frac{\left|\partial_{\mathbf{b}}^{m} F(z)\right|}{m ! L^{m}(z)} \leq \max \left\{\frac{\left|\partial_{\mathbf{b}}^{k} F(z)\right|}{k ! L^{k}(z)}: 0 \leq k \leq m_{0}\right\}
$$

where

$$
\partial_{\mathbf{b}}^{0} F(z)=F(z), \partial_{\mathbf{b}} F(z)=\sum_{j=1}^{n} \frac{\partial F(z)}{\partial z_{j}} b_{j}, \partial_{\mathbf{b}}^{k} F(z)=\partial_{\mathbf{b}}\left(\partial_{\mathbf{b}}^{k-1} F(z)\right), k \geq 2 .
$$

The least such integer $m_{0}=m_{0}(\mathbf{b})$ is called the L-index in the direction $\mathbf{b}$ of the analytic function $F$ and is denoted by $N_{\mathbf{b}}(F, L)=m_{0}$. The definition is a generalization of the concept of bounded $L$-index in direction introduced and considered for entire functions of several variables in $[1,3]$.

The positivity and continuity of the function $L$ and condition (1) are not sufficient to explore the behavior of an analytic function of bounded $L$-index in direction. Below we impose an extra condition that function $L$ does not vary fast. For $\eta \in[0, \beta], z \in \mathbb{B}^{n}$, we define

$$
\begin{gathered}
\lambda_{1}^{\mathbf{b}}(z, \eta, L)=\inf \left\{\frac{L(z+t \mathbf{b})}{L(z)}:|t| \leq \frac{\eta}{L(z)}\right\}, \quad \lambda_{2}^{\mathbf{b}}(z, \eta, L)=\sup \left\{\frac{L(z+t \mathbf{b})}{L(z)}:|t| \leq \frac{\eta}{L(z)}\right\}, \\
\lambda_{1}^{\mathbf{b}}(\eta, L)=\inf \left\{\lambda_{1}^{\mathbf{b}}(z, \eta, L): z \in \mathbb{B}^{n}\right\}, \quad \lambda_{2}^{\mathbf{b}}(\eta, L)=\sup \left\{\lambda_{2}^{\mathbf{b}}(z, \eta, L): z \in \mathbb{B}^{n}\right\} .
\end{gathered}
$$

If it does not cause misunderstanding, then $\lambda_{1}^{\mathbf{b}}(z, \eta) \equiv \lambda_{1}^{\mathbf{b}}(z, \eta, L), \lambda_{2}^{\mathbf{b}}(z, \eta) \equiv \lambda_{2}^{\mathbf{b}}(z, \eta, L)$, $\lambda_{1}^{\mathbf{b}}(\eta) \equiv \lambda_{1}^{\mathbf{b}}(\eta, L), \lambda_{2}^{\mathbf{b}}(z, \eta) \equiv \lambda_{2}^{\mathbf{b}}(\eta, L)$.

By $Q_{\mathbf{b}, \beta}\left(\mathbb{B}^{n}\right)$ we denote the class of all positive functions $L: \mathbb{B}^{n} \rightarrow \mathbb{R}_{+}$satisfying $(1)$ and $0<\lambda_{1}^{\mathbf{b}}(\eta) \leq \lambda_{2}^{\mathbf{b}}(\eta)<+\infty$ for any $\eta \in[0, \beta]$

Definition 1. Analytic function $F: \mathbb{B}^{n} \rightarrow \mathbb{C}$, is said to be of bounded value L-distribution in a direction $\mathbf{b} \in \mathbb{C}^{n} \backslash\{0\}$, if there exist $p \in \mathbb{Z}_{+}$such that for every $w \in \mathbb{C}$ and for all $z_{0} \in \mathbb{B}^{n}$ with $F\left(z^{0}+t \mathbf{b}\right) \not \equiv w$ the equation $F\left(z^{0}+t \mathbf{b}\right)=w$ has in the disc $\left\{t:|t| \leq \frac{1}{L\left(z^{0}\right)}\right\}$ at most $p$ solutions. In other words, the function $F\left(z^{0}+t \mathbf{b}\right)$ is $p$-valent in $\left\{t:|t| \leq \frac{1}{L\left(z^{0}\right)}\right\}$.

Theorem 1. Let $L \in Q_{\mathbf{b}}\left(\mathbb{B}^{n}\right)$. An analytic function $F: \mathbb{B}^{n} \rightarrow \mathbb{C}$ is a function of bounded value $L$-distribution in a direction $\mathbf{b} \in \mathbb{C}^{n} \backslash\{0\}$ if and only if its directional derivative $\frac{\partial F}{\partial \mathbf{b}}$ has bounded $L$-index in the same direction $\mathbf{b}$.

Proof. Assume that the function $F$ is of bounded value $L$-distribution in a direction $\mathbf{b}$, i.e. for every $z^{0} \in \mathbb{B}^{n}$ such that $F\left(z^{0}+t \mathbf{b}\right) \not \equiv$ const the function $F\left(z^{0}+t \mathbf{b}\right)$ is $p$-valent in the $\operatorname{disc}\left\{t:|t| \leq \frac{1}{L\left(z^{0}\right)}\right\}$.

To prove the theorem we need the following proposition [13, p. 48, Theorem 2.8].

Theorem 2 ([13]). Let $D_{0}=\left\{t:\left|t-t_{0}\right|<R\right\}, 0<R<\infty$. If an analytic function in $D_{0}$ is $p$-valent in $D_{0}$ then for $j>p$

$$
\frac{\left|f^{(j)}\left(t_{0}\right)\right|}{j !} R^{j} \leq(A j)^{2 p} \max \left\{\frac{\left|f^{(k)}\left(t_{0}\right)\right|}{k !} R^{k}: 1 \leq k \leq p\right\},
$$

where $A=\sqrt[2 p]{\frac{p+2}{2}} \sqrt{8 e^{\pi^{2}}}$. 
By Theorem 2 inequality (3) holds with $R=\frac{1}{L\left(z^{0}\right)}$ for the function $F\left(z^{0}+t \mathbf{b}\right)$ as a function of one variable $t \in \mathbb{C}$ for every given $z^{0} \in \mathbb{B}^{n}$. For convenience we will use the notation $g_{z^{0}}(t)=F\left(z^{0}+t \mathbf{b}\right)$. Then it is easy to prove that for every $m \in \mathbb{N}$ the following equality $g_{z^{0}}^{(p)}(t)=\frac{\partial^{p} F\left(z^{0}+t \mathbf{b}\right)}{\partial \mathbf{b}^{p}}$ is valid. Put $j=p+1$ and $t_{0}=0$ in Theorem 2. From (3) it follows

$$
\begin{aligned}
& \frac{1}{(p+1) ! L^{p+1}\left(z_{0}\right)}\left|\frac{\partial^{p+1} F\left(z^{0}\right)}{\partial \mathbf{b}^{p+1}}\right| \leq(A(p+1))^{2 p} \max \left\{\frac{1}{k ! L^{k}\left(z_{0}\right)}\left|\frac{\partial^{k} F\left(z^{0}\right)}{\partial \mathbf{b}^{k}}\right|: 1 \leq k \leq p\right\} \Rightarrow \\
& \frac{1}{L^{p+1}\left(z_{0}\right)}\left|\frac{\partial^{p+1} F\left(z^{0}\right)}{\partial \mathbf{b}^{p+1}}\right| \leq(p+1) !(A(p+1))^{2 p} \max \left\{\frac{1}{L^{k}\left(z_{0}\right)}\left|\frac{\partial^{k} F\left(z^{0}\right)}{\partial \mathbf{b}^{k}}\right|: 1 \leq k \leq p\right\} \times \\
& \quad \times \max \left\{\frac{1}{k !}: 1 \leq k \leq p\right\} \Rightarrow \\
& \frac{1}{L^{p}\left(z_{0}\right)}\left|\frac{\partial^{p}}{\partial \mathbf{b}^{p}} \frac{\partial F\left(z^{0}\right)}{\partial \mathbf{b}}\right| \leq L\left(z^{0}\right) \cdot(p+1) ! A^{2 p}(p+1)^{2 p} \max \left\{\frac{1}{L^{k}\left(z_{0}\right)}\left|\frac{\partial^{k-1}}{\partial \mathbf{b}^{k-1}} \frac{\partial F\left(z^{0}\right)}{\partial \mathbf{b}}\right|:\right. \\
& \quad 0 \leq k-1 \leq p-1\} \Rightarrow \frac{\left|\frac{\partial^{p}}{\partial \mathbf{b}^{p}} \frac{\partial F\left(z^{0}\right)}{\partial \mathbf{b}^{p}}\right|}{L^{p}\left(z_{0}\right)} \leq \\
& \leq(p+1) ! A^{2 p}(p+1)^{2 p} \max \left\{\frac{1}{L^{k-1}\left(z_{0}\right)}\left|\frac{\partial^{k-1}}{\partial \mathbf{b}^{k-1}} \frac{\partial F\left(z^{0}\right)}{\partial \mathbf{b}}\right|: 0 \leq k-1 \leq p-1\right\}
\end{aligned}
$$

Now we need an analog of Hayman's Theorem for analytic functions in the unit ball of bounded $L$-index in direction.

Theorem 3 ([5]). Let $L \in Q_{\mathbf{b}}\left(\mathbb{B}^{n}\right)$. An analytic function $F: \mathbb{B}^{n} \rightarrow \mathbb{C}$ has bounded $L$-index in the direction $\mathbf{b}$ if and only if there exist numbers $p \in \mathbb{Z}_{+}$and $C>0$ such that for every $z \in \mathbb{B}^{n}$

$$
\frac{1}{L^{p+1}(z)}\left|\frac{\partial^{p+1} F(z)}{\partial \mathbf{b}^{p+1}}\right| \leq C \max \left\{\frac{1}{L^{k}(z)}\left|\frac{\partial^{k} F(z)}{\partial \mathbf{b}^{k}}\right|: 0 \leq k \leq p\right\}
$$

Thus, for the function $\frac{\partial F}{\partial \mathbf{b}}$ inequality (4) is fulfilled with $p-1$ instead of $p$ and with $C=(p+1) ! A^{2 p}(p+1)^{2 p}$. In Theorem 2 the constant $A \geq \max _{j>p} \frac{p+2}{2}\left(8 e^{\pi^{2}}\right)^{p}\left(1-\frac{1}{j}\right)^{j}$ does not depend on $z^{0}$, because the parameter $p$ is independent of $z^{0}$. Hence, the quantity $C=$ $(p+1) ! A^{2 p}(p+1)^{2 p}$ also does not depend on $z^{0}$. Then by Theorem 3 the function $\frac{\partial F}{\partial \mathbf{b}}$ has bounded $L$-index in the direction $\mathbf{b}$.

On the contrary, let $\frac{\partial F}{\partial \mathbf{b}}$ be an analytic function in the unit ball of bounded $L$-index in the direction $\mathbf{b}$. By Theorem 3 there exist $p \in \mathbb{Z}_{+}$and $C \geq 1$ such that for every $z \in \mathbb{B}^{n}$ the following inequality

$$
\frac{1}{L^{p+1}(z)}\left|\frac{\partial^{p+1} F(z)}{\partial \mathbf{b}^{p+1}}\right| \leq C \max \left\{\frac{1}{L^{k}(z)}\left|\frac{\partial^{k} F(z)}{\partial \mathbf{b}^{k}}\right|: 1 \leq k \leq p\right\}
$$

is true.

Let us consider the $\operatorname{disc} K_{0}=\left\{t \in \mathbb{C}:|t| \leq \frac{1}{L\left(z^{0}\right)}\right\}, z^{0} \in \mathbb{B}^{n}$.

Observe that if $L \in Q_{\mathbf{b}}\left(\mathbb{B}^{n}\right)$, then for all $z^{0} \in \mathbb{B}^{n}, r \in(0, \beta],|t| \leq \frac{r}{L\left(z^{0}\right)}$ the definition of class $Q_{\mathbf{b}}\left(\mathbb{B}^{n}\right)$ implies

$$
\lambda_{1}^{\mathbf{b}}(r) L\left(z^{0}\right) \leq L\left(z^{0}+t \mathbf{b}\right) \leq \lambda_{2}^{\mathbf{b}}(r) L\left(z^{0}\right) .
$$

Now inequalities (5) and (6) for $z=z^{0}+t \mathbf{b}, t \in K$, yield

$$
\frac{1}{(p+1) !}\left|\frac{\partial^{p+1} F\left(z^{0}+t \mathbf{b}\right)}{\partial \mathbf{b}^{p+1}}\right|\left(\frac{1}{C \lambda_{2}^{\mathbf{b}}(1) L\left(z^{0}\right)}\right)^{p+1} \leq
$$




$$
\begin{gathered}
\leq \frac{C p !}{(p+1) !} \max _{1 \leq k \leq p}\left\{\frac{1}{k !}\left|\frac{\partial^{k} F\left(z^{0}+t \mathbf{b}\right)}{\partial \mathbf{b}^{k}}\right|\left(\frac{1}{C \lambda_{2}^{\mathbf{b}}(1) L\left(z^{0}\right)}\right)^{k}\left(\frac{L\left(z^{0}+t \mathbf{b}\right)}{C \lambda_{2}^{\mathbf{b}}(1) L\left(z^{0}\right)}\right)^{p+1-k}\right\} \leq \\
\leq \frac{C}{p+1} \max _{1 \leq k \leq p}\left\{\frac{1}{k !}\left|\frac{\partial^{k} F\left(z^{0}+t \mathbf{b}\right)}{\partial \mathbf{b}^{k}}\right|\left(\frac{1}{C \lambda_{2}^{\mathbf{b}}(1) L\left(z^{0}\right)}\right)^{k}\left(\frac{1}{C}\right)^{p+1-k}\right\} \leq \\
\leq \max _{1 \leq k \leq p}\left\{\frac{1}{k !}\left|\frac{\partial^{k} F\left(z^{0}+t \mathbf{b}\right)}{\partial \mathbf{b}^{k}}\right|\left(\frac{1}{C \lambda_{2}^{\mathbf{b}}(1) L\left(z^{0}\right)}\right)^{k}\right\} .
\end{gathered}
$$

To complete the proof of the theorem we will apply the following proposition from $[13, \mathrm{p} .44$, Theorem 2.7].

Theorem 4 ([13, p.44, Theorem 2.7], [8]). Let $D_{0}=\left\{t \in \mathbb{C}:\left|t-t_{0}\right|<R\right\}, 0<R<+\infty$, and $f$ be an analytic function in $D_{0}$. If for all $z \in D_{0}$

$$
\left(\frac{R}{2}\right)^{p+1} \frac{\left|f^{(p+1)}(t)\right|}{(p+1) !} \leq \max \left\{\left(\frac{R}{2}\right)^{k} \frac{\left|f^{(k)}(z)\right|}{k !}: 1 \leq k \leq p\right\},
$$

then $f$ is $p$-valent in $\left\{t \in \mathbb{C}:\left|t-t_{0}\right| \leq \frac{R}{25 \sqrt{p+1}}\right\}$, i. e. the function $f(t)$ attains each value at most $p$ times.

Inequality (7) implies estimate (8) with $R=\frac{2}{C \lambda_{2}^{\mathbf{b}}(1) L\left(z^{0}\right)}$ for $t_{0}=0$. By Theorem 4 the function $F\left(z^{0}+t \mathbf{b}\right)$ is $p$-valent in the disc $\left\{t \in \mathbb{C}:|t| \leq \frac{\rho}{L\left(z^{0}\right)}\right\}, \rho=\frac{2}{25 C \lambda_{2}^{\mathrm{b}}(1) \sqrt{p+1}}$.

Let $t_{j}$ be an arbitrary point in $K_{0}$ and $K_{j}^{*}=\left\{t \in \mathbb{C}:\left|t-t_{i}\right| \leq \frac{\rho^{2}}{L\left(z^{0}+t_{j} \mathbf{b}\right)}\right\}$. Since, by the definition of class $Q_{\mathbf{b}}\left(\mathbb{B}^{n}\right), L\left(z^{0}+t_{j} \mathbf{b}\right) \leq \lambda_{2}^{\mathbf{b}}(1) L\left(z^{0}\right)$, we have that

$$
K_{j}=\left\{t \in \mathbb{C}:\left|t-t_{j}\right| \leq \frac{\rho}{\lambda_{2}^{\mathbf{b}}(1) L\left(z^{0}\right)}\right\} \subset K_{j}^{*}
$$

We can repeat the above arguments for the set $\left\{t \in \mathbb{C}:\left|t-t_{j}\right| \leq \frac{1}{L\left(z^{0}+t_{j} \mathbf{b}\right)}\right\}$ to obtain that the function $F\left(z^{0}+t \mathbf{b}\right)$ is $p$-valent in $K_{j}^{*}$. Since $K_{j} \subset K_{j}^{*}$, the function $F\left(z^{0}+t \mathbf{b}\right)$ is $p$-valent in $K_{j}$.

Finally, we remark that every closed disc of radius $R_{*}$ can be covered by a finite number $m_{*}$ of closed discs of radius $\rho_{*}<R_{*}$ with centers in this disc. Moreover, $m_{*}<B_{*}\left(R_{*} / \rho_{*}\right)^{2}$, where $B_{*}>0$ is a constant. Hence, we can cover the set $K_{0}$ by a finite number $m$ of discs $K_{j}$, where $m \leq 625 B^{*}(p+1) C^{2}\left(\lambda_{2}^{\mathbf{b}}(1)\right)^{2} / 4$. Since the function $F\left(z^{0}+t \mathbf{b}\right)$ in $K_{j}$ is $p$-valent, it is $m p$-valent in $K_{0}$.

In view of arbitrarity of $z^{0}$, the theorem is proved.

\section{REFERENCES}

1. A.I. Bandura, O.B. Skaskiv, Directional logarithmic derivative and the distribution of zeros of an entire function of bounded L-index along the direction, Ukrain. Mat. J., 69 (2017), №3, 500-508.

2. A. Bandura, O. Skaskiv, Functions analytic in a unit ball of bounded L-index in joint variables, J. Math. Sci., 227 (2017), №1, 1-12.

3. A. Bandura, O. Skaskiv, Entire functions of several variables of bounded index, Lviv: Publisher I.E. Chyzhykov, 2016, 128 p. 
4. A.I. Bandura, The properties of entire functions of bounded value L-distribution in direction Prykarp. Visn. Nauk. Tov. Im. Shevchenka, 1(13) (2011), 50-55.

5. A. Bandura, O. Skaskiv, Analytic in the unit ball functions of bounded L-index in direction, https://arxiv.org/abs/1501.04166

6. G.H. Fricke, A note on bounded index and bounded value distribution, Indian J. Pure Appl. Math., 11 (1980), №4, 428-432.

7. G.H. Fricke, S.M. Shah, On bounded value distribution and bounded index, Nonlinear Anal., 2 (1978) №4, 423-435.

8. W.K. Hayman, Differential inequalities and local valency, Pacific J. Math., 44 (1973) №1, 117-137.

9. A.D. Kuzyk, M.N. Sheremeta, Entire functions of bounded l-distribution of values, Math. Notes, 39 (1986), №1, 3-8.

10. B. Lepson, Differential equations of infinite order, hyperdirichlet series and entire functions of bounded index, Proc. Sympos. Pure Math., Amer. Math. Soc.: Providence, Rhode Island, 2 (1968), 298-307.

11. R. Roy, S.M. Shah, The product of two functions of bounded value distribution, Indian J. Pure Appl. Math., 17 (1986), №5, 690-693.

12. R. Roy, S.M. Shah, Functions of bounded index, bounded value distribution and v-bounded index, Nonlinear Anal., 11 (1987), 1383-1390.

13. M. Sheremeta, Analytic functions of bounded index, Lviv: VNTL Publishers, 1999, $141 \mathrm{p}$.

14. S. Shah, Entire functions of bounded value distribution and gap power series In: P. Erdôs, L. Alpár, G. Halász, A. Sárközy (eds.) Studies in Pure Mathematics To the Memory of Paul Turán, pp. 629-634, Birkhäuser Basel, 1983.

15. M.N. Sheremeta, An l-index and an l-distribution of the values of entire functions, Soviet Math. (Iz. VUZ), 34 (1990), №2, 115-117.

Ivano-Frankivsk National Technical University of Oil and Gas

Ivano-Frankivsk, Ukraine

andriykopanytsia@gmail.com 